Scientific paper

\title{
Electrochemical Circuit Elements
}

\author{
Joachim Maier \\ Max Planck Institute for Solid State Research, Heisenbergstr. 1, 70569 Stuttgart, Germany \\ * Corresponding author: E-mail: s.weiglein@fkf.mpg.de
}

Received: 04-03-2016

In memory of prof. dr. Janko Jamnik.

\begin{abstract}
The vast majority of electrochemical processes can be modelled by resistors and capacitors. These will then be, in addition to usual circuit elements, electrochemical and chemical resistors or chemical capacitors. The paper shows the significance of understanding these parameters and their connections in given systems for a variety of timely scientific examples. This rationale mirrors one of the intellectual facets, if not the most important one, of Janko Jamnik's scientific work.
\end{abstract}

Keywords: Equivalent circuit, chemical capacitor, electrochemical resistor, transport, batteries, fuel cells

\section{Introduction}

This contribution is a tribute to Janko Jamnik's outstanding comprehension of electrochemical processes. In many joint papers we set out the concept of analyzing processes in terms of electrochemical equivalent circuits, by not only including electrical but also chemical and electrochemical circuit elements. ${ }^{1-5}$ Although there have been earlier treatments, ${ }^{6-10}$ the above-mentioned publications provide a significant step forward in terms of interpretation and understanding.

The set-out conception allows one to intuitively, but rather precisely though, tackle diverse problems such as stoichiometric polarization, Maxwell-Wagner polarization, battery storage, chemical diffusion in heterogeneous systems, or surface kinetics of oxygen incorporation in oxides.

A central role is played by the archetypical chemical capacitance that allows for a profound understanding of solid state processes whenever concentration changes are involved. The term has already been introduced by Pelton. ${ }^{11}$ Here a more appropriate thermodynamic access will be given. For this purpose we consider a binary solid (generalization is trivial), where the composition of mobile component is regulated by fixing the chemical potential of component $1 \mu_{1}$ (e.g. by outer partial pressure), while the rigid component 2 is characterized by a given mole number $n_{2}$. The other control parameters are total pressure $(p)$ and temperature $(T)$. (Note that it is possible to vary the partial pressure of component 1 at constant total pressu- re.) One then refers to a $\mu_{1} N_{2} p T$-ensemble for which the characteristic potential will be

$$
\Gamma=U-T S+p V-\mu_{1} n_{1}
$$

with the consequence that

$$
\begin{aligned}
d \Gamma & =V d p-S d T+\mu_{2} d n_{2}-n_{1} d \mu_{1} \\
& =V d p-S d T-n_{1} d \mu_{1} \equiv \Sigma_{i} \ell_{i} d \lambda_{i} .
\end{aligned}
$$

(As the generalized Gibbs-Duhem equation leads, for homogeneous systems, to $U=T S-p V+\mu_{1} n_{1}+\mu_{2} n_{2}$, one finds $\Gamma=\mu_{2} n_{2}$. For a one-component system, it results that $\Gamma=0$, corresponding to the characteristics of the "intensive ensemble" referred to. ${ }^{12}$ )

We can now define generalized capacities via $\frac{\partial^{2} \Gamma}{\partial \lambda_{i}^{2}}$, namely the thermal capacitances (specific heat $C^{T} \equiv T \frac{\partial S}{\partial T}$ $=-T \frac{\partial^{2} \Gamma}{\partial T^{2}}$ ), the mechanical capacitance (compressibility $\left.\chi \equiv-\frac{1}{V} \frac{\partial V}{\partial p}=-\frac{1}{V} \frac{\partial^{2} \Gamma}{\partial p^{2}}\right)$ and the chemical capacitance, viz. $C_{1}^{\delta} \equiv \frac{\partial n_{1}}{\partial \mu_{1}}=-\frac{\partial^{2} \Gamma}{\partial \mu_{1}^{2}}$. The sign of these three quantities is decisive in a thermodynamic stability analysis. ${ }^{13}$ One realizes that the definition is also analogous to the electrical capacitance $C^{q}=\frac{\partial q}{\partial \phi} \quad(q$ : charge, $\phi$ : electric potential $)$ that 
becomes relevant if one also includes electrical effects. In view of the significance of the electrochemical potential $\tilde{\mu}=\mu+z F \phi$ one is tempted to define a generalized electrochemical capacitance $\tilde{C}_{1}^{\delta}=\frac{\partial n_{1}}{\partial \tilde{\mu}_{1}}$. Yet as shown in Ref. [5] this is only meaningful in very special cases. In general $C^{\delta}$ and $C^{q}$ refer to different microscopic processes. In contrast, the introduction of an electrochemical resistor $R^{\delta}$ (in the quasi-one dimensional case defined via $\nabla \tilde{\mu} / j$ ), however, is generally meaningful owing to current density $j$ being proportional to $\sigma \nabla \tilde{\mu}=\sigma \nabla \mu+z F \sigma \nabla \phi$, i.e., owing to referring to the very same transport process caused by both concentration and electrical potential gradients $(\sigma$ : conductivity). Let us consider a few selected examples.

\section{Chemical Diffusion}

A comparatively simple situation is chemical diffusion in the bulk, e.g. describing the variation of oxygen stoichiometry on varying the outside oxygen partial pressure. The diffusion coefficient describing that variation is

$$
D^{\delta} \propto\left(\frac{\sigma_{\text {ion }} \sigma_{\text {en }}}{\sigma}\right)\left(\frac{\partial \mu_{\mathrm{O}}}{\partial c_{\mathrm{O}}} \cdot \frac{1}{R T}\right) .
$$

For a simple planar geometry ( $L$ : thickness, $A$ : area) the first factor can be rewritten as inverse chemical resistor

$$
R^{\delta}=\frac{\sigma}{\sigma_{\text {ion }} \sigma_{\text {eon }}} \frac{L}{A},
$$

resulting from a series switching of ionic and electronic resistors, as in a logical sense both ions and electrons are needed. ${ }^{14}$

The second factor in Eq. (3) can be rewritten as $\left(\frac{\partial \ln a_{\mathrm{O}}}{\partial \ln c_{\mathrm{O}}} \frac{1}{c_{\mathrm{O}}}\right)$, where $a_{\mathrm{O}}$ is the oxygen activity and $\frac{\partial \ln a_{\mathrm{O}}}{\partial \ln c_{\mathrm{O}}}$ is referred to as thermodynamic factor, often erroneously termed an enhancement factor. In fact the second factor represents an inverse chemical capacitance ${ }^{15}$

$$
C^{\delta}=A \cdot L \frac{\partial c_{\mathrm{O}}}{\partial \mu_{\mathrm{O}}} \frac{R T}{F^{2}}
$$

expressing the storage effect, here occurring in the course of bulk diffusion. The term enhancement factor stems from the incorrect conception of comparing $D^{\delta}$ with the tracer diffusion coefficient $D^{*}$. Yet, chemical diffusion refers to the defects only, and a closer look yields under dilute conditions $\left(\frac{1}{c_{\text {ion }}}+\frac{1}{c_{\text {eon }}}\right)$ for the second term, with $c_{\text {ion }}$, $c_{\text {eon }}$ being the respective defect concentrations. Tracer diffusion, however, involves the total ionic ensemble and has hence a much higher chemical capacitance, thus $D^{*}$ must be much smaller owing to the low fraction of defects. The fair comparison is with the defect diffusion coefficients. The analogously defined thermodynamic factor of the defects is now close to unity and in most cases even reflects a depression factor. ${ }^{16}$

The product $R^{\delta} \cdot C^{\delta}$ yields the time constant $\tau^{\delta}$ as $L^{2} / D^{\delta}$, which is well-known from diffusion kinetics. (Depending on the boundary conditions, the relation between $\tau^{\delta}$ and $L^{2} / D^{\delta}$ includes numerical constants.) At any rate $\tau^{\delta}$ is proportional to $L^{2}$ as both $R^{\delta}$ and $C^{\delta}$ are proportional to $L$. This is very different from the electric analogue where for quasi-1D transport (along $L$ ) $R$ $\propto L$ but $C \propto 1 / L$ with $\tau=R C$ being independent of $L$. Table I gives thickness dependences of various time constants for transport along $L$ for various degrees of nano-structuring: nano-plates of thickness $L$ (dimensionality of nano-structuring $d=1$ ), nano-rods of cross section $L \times L$ (i.e. $d=2$ ) and nanodots of dimension $L \times$ $L \times L$ (i.e. $d=3$ ), showing that the results for $\tau_{\varepsilon}$ are independent of $d .^{17}$

Table I: Dimensionality $(d)$ dependent exponents $(n)$ of the explicit thickness $(L)$ dependence of resistors, capacitors, relaxation times for various electrochemical mechanisms $(\varepsilon) .{ }^{17}$ The interface process is in series with bulk process, i.e. transport across the boundary is considered.

\begin{tabular}{lccc}
\hline mechanism $\boldsymbol{\varepsilon}$ & $\mathbf{R}_{\boldsymbol{\varepsilon}}$ & $\mathbf{C}_{\boldsymbol{\varepsilon}}$ & $\boldsymbol{\tau}_{\boldsymbol{\varepsilon}}$ \\
\hline $\begin{array}{l}\text { dielectric response } \\
\text { diffusion controlled bulk } \\
\text { storage }\end{array}$ & $2-d$ & $d-2$ & 0 \\
$\begin{array}{l}\text { interfacial rate controlled } \\
\text { bulk storage }\end{array}$ & $2-d$ & $d$ & 2 \\
$\begin{array}{l}\text { interfacial storage } \\
(\text { semi-infinite) }\end{array}$ & $1-d$ & $d$ & 1 \\
\hline
\end{tabular}

\footnotetext{
${ }^{\#}$ In the finite size regime the situation is non-autonomous as it also depends on the neighboring phases. Reprinted with permission from the American Chemical Society, Copyright 2013. ${ }^{17}$
}

The generalization of the chemical diffusion concept to internal reactions includes so-called differential trapping factors. ${ }^{18}$ This generalization nicely explains why $D^{\delta}$ in Fe-doped $\mathrm{SrTiO}_{3}$ is orders of magnitude smaller than expected according to the then established theory. The reason is the coupling of internal redox reactions to the electronic ensemble. The same happens for Y-doped $\mathrm{ZrO}_{2}$, where both ionic and electronic conductivities are insensitive to additional redox active impurities. ${ }^{19}$ An intuitive understanding again is enabled by the concept of chemical capacitance. In $\mathrm{ZrO}_{2}$ the internal valence changes do not vary $R^{\delta}$ but increase the chemical capacitance (increase of reservoir). 


\section{Interfacial Kinetics}

The chemical capacitance concept became most influential as far as the surface kinetic analysis is concerned. Here $R^{\delta}$ refers to the reaction rate of the rate-limiting surface step, but $C^{\delta}$ is given by the uptake in the bulk as long as the sample is not extremely thin. The corresponding equivalent circuit is now used in labs all over the world to analyze surface kinetics via impedance spectroscopy (cf. Fig. 1) (cf. Ref. ${ }^{20}$ for a recent example of interest). In most cases of interest, the decisive resistive term refers to the surface kinetics, while the capacitive terms refer to the bulk capacity
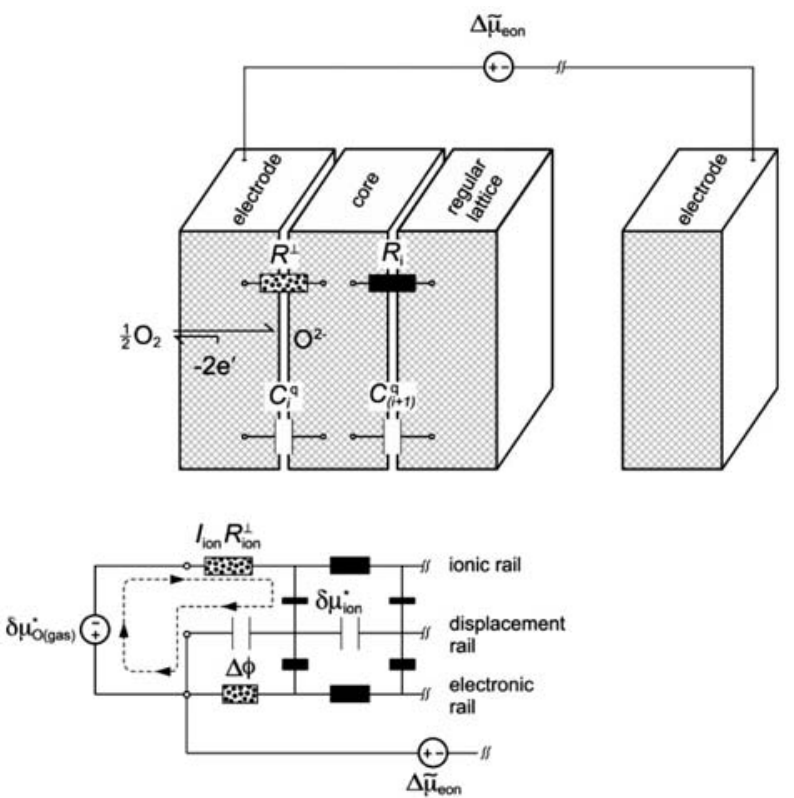

Figure 1. Equivalent circuit of the electrode/electrolyte impedance under gas exchange. Reprinted by permission of the PCCP Owner Societies. $^{5}$

A further highly relevant example is mass transport across a grain boundary. Then $R^{\delta}$ refers to the transfer resistance of the neutral mass, typically controlled by the structure of the grain boundary core or by space charge effects owing to depletion. Well investigated are grain boundaries in $\mathrm{SrTiO}_{3}$, where both necessary defects, the oxygen as well as electronic defects are depleted and hence $R^{\delta}$ is substantial. Again $C^{\delta}$ refers to the bulk if one can ignore storage of oxygen in the grain boundary itself. The detailed treatment of transport across space charge zones is rather involved. ${ }^{1}$

The full power of these considerations unfolds in heterogeneous systems (Fig. 2).

If we refer to a polycrystal in which grain boundary diffusion is very fast so that transport from there to the grains of size $\ell$ occurs on a different time scale, both $R^{\delta}$ and $C^{\delta}$ in the effective $D_{m}^{\delta}$ are due to bulk, ${ }^{16}$ and hence

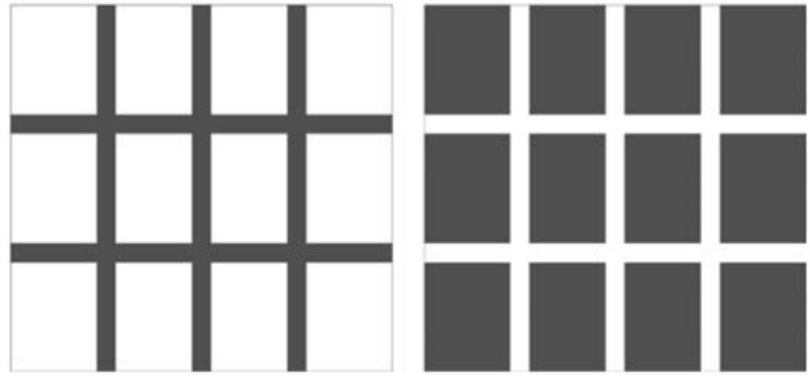

Figure 2. Diffusion through a ceramic with highly permeable grain boundaries (left) or scarcely permeable grain boundaries (right). The black and white zones refer to the local permeabilities.

$$
D_{m}^{\delta}=\frac{L^{2}}{\ell^{2}} D_{\text {bulk }}^{\delta} \propto \frac{L^{2}}{\ell^{2}} \frac{1}{R_{\text {bulk }}^{\delta} C_{\text {bulk }}^{\delta}} .
$$

Such a situation is, e.g., met in the case of chemical diffusion in donor-doped $\mathrm{SrTiO}_{3}$. In the opposite case that the grain boundaries of width $d_{\mathrm{gb}}$ are hardly permeable and transport through them is very sluggish when compared to the bulk, a case briefly addressed above, it holds for not too small grains. ${ }^{16,21}$

$$
D_{m}^{\delta} \propto \frac{\ell}{d_{\mathrm{gb}}} \frac{1}{R_{\mathrm{gb}}^{\delta} C_{\mathrm{bulk}}^{\delta}} .
$$

Even though redistribution in the grain interior is locally fast, grain size matters for the time behavior, as the chemical diffusion is the more sluggish, the more component mass the grain-interior is able to take up.

In fact generally the diffusion rate is not only determined by the rates at which the carriers would move in the steady state $\left(R^{\delta}\right)$ but also by how much mass is absorbed $\left(C^{\delta}\right)$. This is analogous to thermal diffusion, where the speed of the temperature variation not only depends on the thermal conductivity (conduction of heat) but also on the heat capacity (absorption of heat).

At the moment we are considering chemical diffusion along interfaces where again deconvolution into $R^{\delta}$ and $C^{\delta}$ is highly beneficial. It seems that chemical diffusion in job-sharing composites along the boundaries is extremely fast not only due to the expectedly low $R^{\delta}$ but also due to a very low $C^{\delta}$. Here one meets one of the cases in which $C^{\delta}$ represents truly an electrochemical capacitance. $^{22}$

\section{Impedance of Mixed Conductor}

A long-standing impedance problem became transparent by the use of equivalent circuits. ${ }^{23}$ Imagine a purely ionic conductor between electrodes that are only reversible for electrons. Evidently, the material should behave as a capacitor with vertical line $\left(90^{\circ}\right)$ in the impedance plot. Now let us tackle this problem from the viewpoint of 
a mixed conductor with vanishing electronic conductivity. For a mixed conductor one expects a Warburg increase $\left(45^{\circ}\right)$ bending in a semi-circular behavior and an intercept yielding the electronic resistance. If one nullifies the electronic contribution (i.e. pure ion conductor) an inconsistency occurs as we would obtain an infinitely extended Warburg line and not a vertical one.

This inconsistency is shown to be due to the neglect of space charge polarization that also must occur. The generalized approach is characterized by the counter-play of space charge and chemical bulk capacitances depending on the charge carrier concentration rather than the conductivities (Fig. 3). ${ }^{23}$

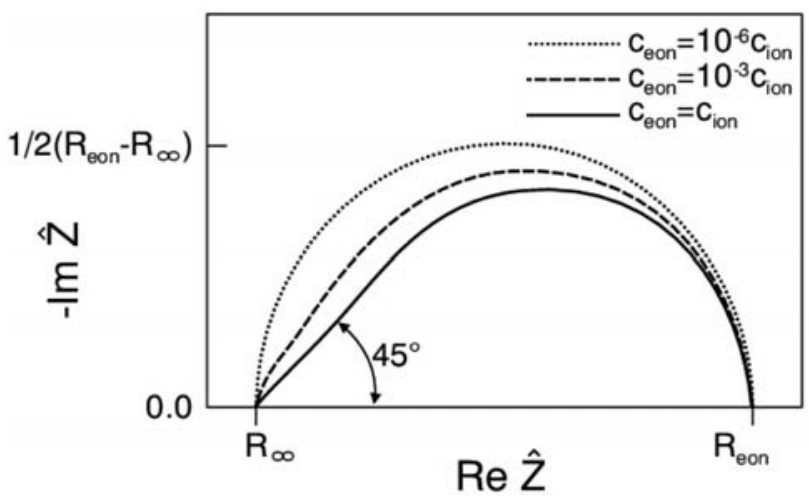

Figure 3. Normalized impedances for ion blockage. On variation of the defect concentration the response changes from Warburg to a pure semicircular behavior. Reprinted with permission from Elsevier. $^{23}$

Other contributions to be mentioned in that context are (i) the polarization behavior of a polycrystalline material in which the grain boundaries as well as electrodes can lead to a stoichiometric polarization ${ }^{24}$ and (ii) the establishing of a penetration impedance method with the potential of identifying buried interfaces. ${ }^{25}$

\section{System Impedance and Relevance of Morphology}

As already mentioned, electrochemical processes of interest can be modelled by $R$ 's and $C$ 's if we refer to electrical, chemical and electrochemical circuit elements. Figure 4 indicates that size dependencies can occur through size dependencies of the effective materials constants, e.g., overall conductivities of a composite (e.g. through space charge effects), but also through the path dependence itself ( $\mathrm{n}=$ 2 for chemical diffusion, see above). This points towards the necessity for electrochemically integrated circuits for high performance electrode design in Li-batteries. ${ }^{17}$

Figure 5 shows the equivalent circuit of a battery as given by Jamnik. It clearly exhibits that both the ionic path $\left(\mathrm{Li}^{+}(\mathrm{Li}) \rightarrow \mathrm{Li}^{+}\right.$(electrolyte) $\rightarrow \mathrm{Li}^{+}$(counter electro-

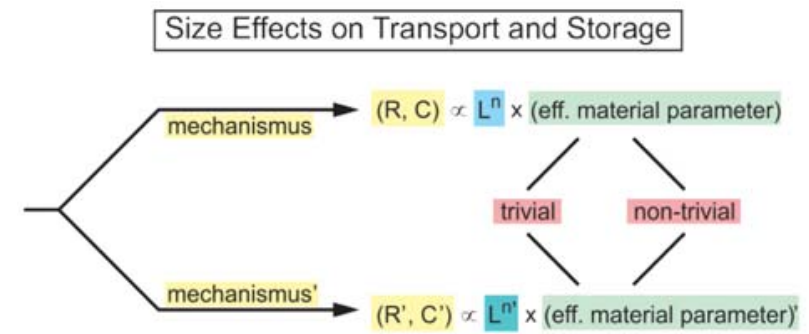

Figure 4. Explicit and implicit size dependence of resistive and capacitive elements. The explicit size dependence $\left(\mathrm{L}^{n}\right)$ reflects the direct geometrical influence (cf. Table 1), the implicit size dependence mirrors the dependence of the effective materials parameter on the interfacial density in the case of a heterogeneous object. ${ }^{17} \mathrm{Tu}-$ ning the parameters by size effects can even result in a switchingover to an alternative mechanism. Reprinted with permission from the American Chemical Society, Copyright 2013. ${ }^{17}$

de) $\rightarrow \|$ current collector) and the electronic path ( $e^{-}$(current collector $) \rightarrow e^{-}$(electrode) $\rightarrow \|$ (electrolyte) $)$ are stopped by chemical capacitors.
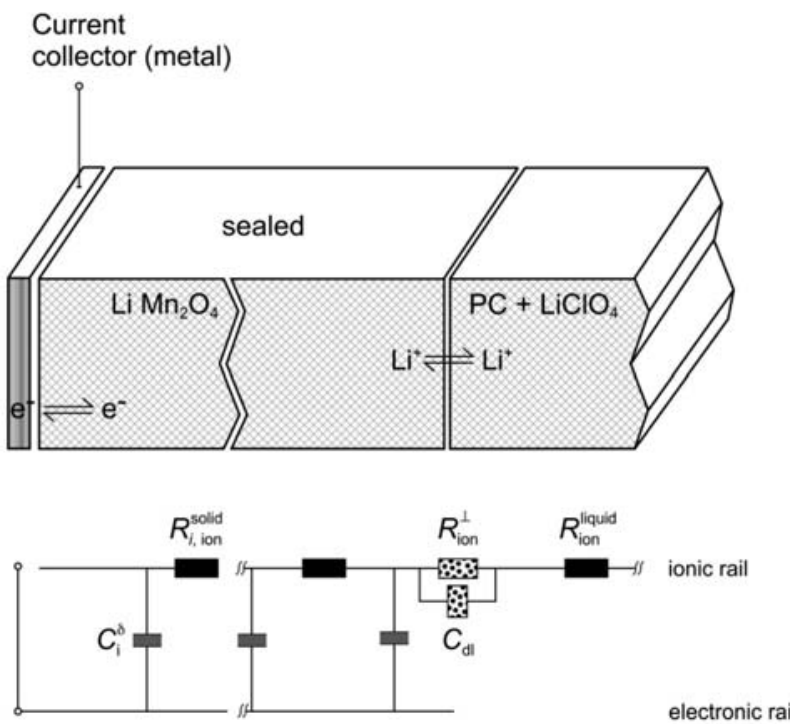

Figure 5. Scheme and equivalent circuit of a lithium battery (cathode side). Reprinted by permission of the PCCP Owner Societies. ${ }^{5}$

This equivalent circuit can describe the mass and charge variation upon discharge/charge in quite a detail. Solid state electrochemistry is full of such interwoven electrical and chemical problems and in such cases Jamnik's contributions are of invaluable worth and we miss him as a competent discussion partner.

\section{References}

1. J. Jamnik and J. Maier, Ber. Bunsenges. Phys. Chem., 1997, 101, 23-40. 
http://dx.doi.org/10.1002/bbpc.19971010104

2. J. Jamnik and J. Maier, J. Phys. Chem. Solids, 1998, 59, 1555-1569.

http://dx.doi.org/10.1016/S0022-3697(98)00065-1

3. J. Jamnik, J. Maier and S. Pejovnik, Electrochim. Acta, 1999, 44, 4139-4145.

http://dx.doi.org/10.1016/S0013-4686(99)00128-0

4. J. Jamnik and J. Maier, J. Electrochem. Soc., 1999, 146, 4183-4188. http://dx.doi.org/10.1149/1.1392611

5. J. Jamnik and J. Maier, Phys. Chem. Chem. Phys., 2001, 3, 1668-1678. http://dx.doi.org/10.1039/b100180i

6. C. T. Sah, Solid State Electron.,1970, 13, 1547-1575. http://dx.doi.org/10.1016/0038-1101(70)90035-3

7. G. C. Barker, J. Electroanal. Chem., 1973, 41, 201-211. http://dx.doi.org/10.1016/S0022-0728(73)80438-3

8. J. Maier, Z. Phys. Chem. NF, 1984, 140, 191-215. http://dx.doi.org/10.1524/zpch.1984.140.2.191

9. D. R. Franceschetti, Solid State Ionics, 1994, 70/71, 542547. http://dx.doi.org/10.1016/0167-2738(94)90369-7

10. R. P. Buck and C. Mundt, Electrochim. Acta, 1999, 44, 1999-2018.

http://dx.doi.org/10.1016/S0013-4686(98)00309-0

11. A. D. Pelton, J. Chim. Phys., 1992, 89, 1931-1949.

12. J. E. Kirkpatrick, Statistical Mechanics, in Physical Chemistry. An Advanced Treatise, Vol. II, H. Eyring, D. Henderson, W. Jost (eds.), Academic Press, New York, 1971.

13. A. Sanfeld, in: Physical Chemistry, An Advanced Treatise, Vol. I, Thermodynamics, H. Eyring, D. Henderson, W. Jost (eds.), p. 245, Academic Press, New York, 1971.
14. C. Wagner, Prog. Solid St. Chem., 1975, 10, 3-16. http://dx.doi.org/10.1016/0079-6786(75)90002-3

15. J. Maier, Solid State Phenom.,1994, 39-40, 35-60. http://dx.doi.org/10.4028/www.scientific.net/SSP.39-40.35

16. J Maier, Physical Chemistry of Ionic Materials. Ions and Electrons in Solids, John Wiley \& Sons, Ltd, Chichester, UK, 2004. http://dx.doi.org/10.1002/0470020229

17. J. Maier, Chem. Mater, 2014, 26, 348-360. http://dx.doi.org/10.1021/cm4021657

18. J. Maier, J. Am. Ceram. Soc., 1993, 76, 1212-1217. http://dx.doi.org/10.1111/j.1151-2916.1993.tb03743.x

19. K. Sasaki and J. Maier, Solid State Ionics, 2000, 134, 303321. http://dx.doi.org/10.1016/S0167-2738(00)00766-9

20. D. Poetzsch, R. Merkle and J. Maier, J. Electrochem. Soc., 2015, 162, F939-F950. http://dx.doi.org/10.1149/2.0951508jes

21. J. Jamnik, in: Solid State Ionics: Science \& Technology, B. V. R. Chowdari, K. Lal,S. A. Agnihotry, N. Khare, S. S. Sekhon, P. C. Srivastava, S. Chandra (eds.), p. 13, World Scientific Publishing Co., Singapore, 1998.

22. C.-C. Chen and J. Maier, to be published.

23. J. Jamnik and J. Maier, J. Electrochem. Soc., 1999, 146, 4183-4188. http://dx.doi.org/10.1149/1.1392611

24. J. Jamnik, X.Guo and J. Maier, Appl. Phys. Lett., 2003, 82, 2820-2822. http://dx.doi.org/10.1063/1.1570513

25. J. Jamnik, J. Maier and S. Pejovnik, Electrochim. Acta, 1996, 41, 1011-1015. http://dx.doi.org/10.1016/0013-4686(95)00432-7

\section{Povzetek}

Večino elektrokemijskih procesov lahko modelno predstavimo z upori in kondenzatorji. Ti bodo nato poleg običajnih elementov vezja, elektrokemijski in kemijski upori ali kemijski kondenzatorji. V članku je prikazan pomen razumevanja teh parametrov in njihovih povezav v določenih sistemih, za različne aktualne znanstvene primere. To načelo se zrcali v enem izmed intelektualnih pristopov, ki so znanstveno delo, morda celo najpomembnejše, Janka Jamnika. 